\title{
Malignant melanoma representing with testicular metastasis: a case report and review of the literature
}

\author{
Muhammad Z. Aslam, MD; M.S. Ahmed, MD; Sri Nagarajan, MD; Syed T. Rizvi, MD
}

\section{Abstract}

We present the case of a 52-year-old man, with a history of malignant melanoma, who presented with a testicular lump. Radical orchidectomy confirmed a metastatic malignant melanoma. We discuss salient features of this disease in the light of the published literature.

Can Urol Assoc J 2010;4(4):E103-104

\section{Case report}

A 52-year-old man presented to our department with a 2 -week history of pain and swelling in the left testis. Clinical examination revealed hard irregular consistency of the whole testis with slight tenderness. Ultrasonography confirmed complete obliteration of the left testis by a mixed echogenic lesion with areas of necrosis consistent with malignancy. Testicular tumour markers were within normal range.

He had a history of cutaneous malignant melanoma involving the left forearm and underwent wide local excision 2 years before his current presentation. Excision biopsy had confirmed a 2.1-mm Breslow thickness malignant melanoma.

A left inguinal orchidectomy was performed. On histopathology, the tumour had a multinodular appearance, composed of atypical oval to spindle-shaped cells with several mitotic figures, very focal intracytoplasmic melanin pigment deposition and interspersed lymphocytes - these features confirmed the diagnosis of an amelanotic metastatic malignant melanoma. The immunohistochemical profile further supported the diagnosis (Fig. 1). A staging computed tomography (CT) scan postoperatively confirmed widespread bilateral pulmonary nodules representative of metastatic disease (Fig. 2).

Our patient was referred to oncologists for chemotherapy; they initially decided to have a period of observation with interval CT scans. The patient re-presented 12 months later with seizures. A CT scan had confirmed widespread cerebral metastasis (Fig. 3). The patient required admission to the intensive treatment unit and died soon afterwards.

\section{Discussion}

Metastatic carcinoma to the testis is a rare finding and may simulate primary testicular neoplasms. The most common primary site has been reported to be the prostate gland, contributing $35 \%{ }^{1}$ and $38 \%{ }^{2}$ of all primaries in 2 separate series. Other common sites include the lung, colon and kidney.

Malignant melanoma metastasizing to the testis is among the most aggressive and life-threatening tumours and are usually found during autopsy. ${ }^{3,4}$ The incidence of cutaneous malignant melanoma as the primary site in metastatic testicular tumours has been reported to vary between $9 \%{ }^{1}$ and $41 \%{ }^{4}$

The most common presentation is a rapidly growing testicular mass. 5,6,7 Patients have also been reported to present with melanospermia ${ }^{7}$ and with supraclavicular mass, the biopsy of which had shown lymph node metastasis. ${ }^{8}$

The primary testicular tumour is known to be a disease of young men in their 20s and 30s, but the presentation of testicular metastasis of malignant melanoma varied widely between 43 to 80 years. ${ }^{5,6}$ Despite the late presenting age, it is of immense importance to exclude primary disease as it is far more common than metastatic tumour. Unlike the primary disease, serum testicular tumour markers were not found to be elevated.5,6,7 Although a definitive diagnosis could only be established after histopathological analysis, a careful history can help clinicians anticipate the diagnosis, as most cases in the literature were known to have a history of cutaneuos primary disease. No mechanism for melanoma metastasis to testis has been established to date. ${ }^{9}$

The prognosis is reported to be very poor in these patients. In most cases, the interval between orchidectomy and death is reported to be less than 12 months. ${ }^{5,6,7}$

\section{Conclusion}

Malignant melanoma metastasising to testis is a rare, but aggressive, disease with a very poor prognosis and a very high mortality rate within a short interval. We recommend early intervention and oncological involvement in these patients to improve survival. 


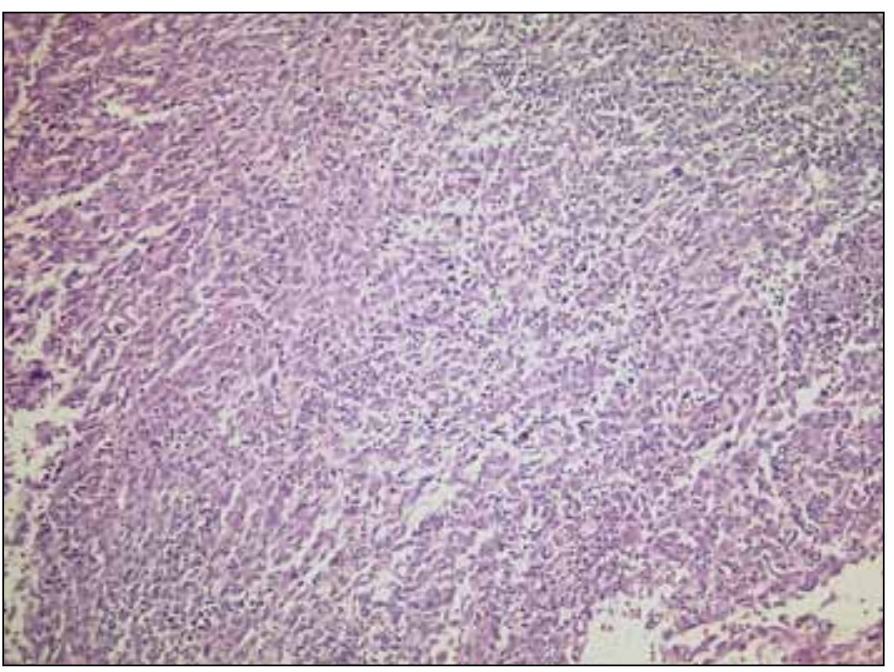

Fig. 1. Histopathological features consistent with an amelanotic metastatic malignant melanoma.

Department of Urology, South Tees Hospitals, United Kingdom

Competing interests: None declared.

This paper has been peer-reviewed.

\section{References}

1. Haupt HM, Mann RB, Trump DL, et al. Metastatic carcinoma involving the testis. Clinical and pathologic distinction from primary testicular neoplasma. Cancer 1984;54:709-14.

2. Ulbright T, Young RH. Metastatic carcinoma to the testis. A clinicopathological analysis of 26 nonincidental cases with emphasis on deceptive features. Am J Surg Pathol 2008:32:1683-93.

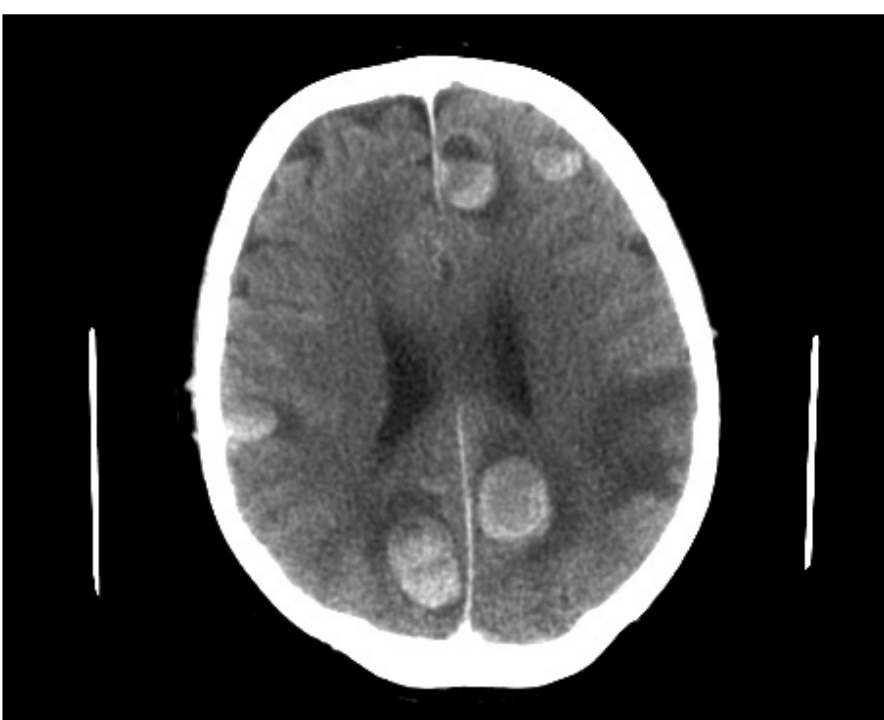

Fig. 3. Cerebral metastasis demonstrated on computed tomography scan performed 18 months postoperatively.

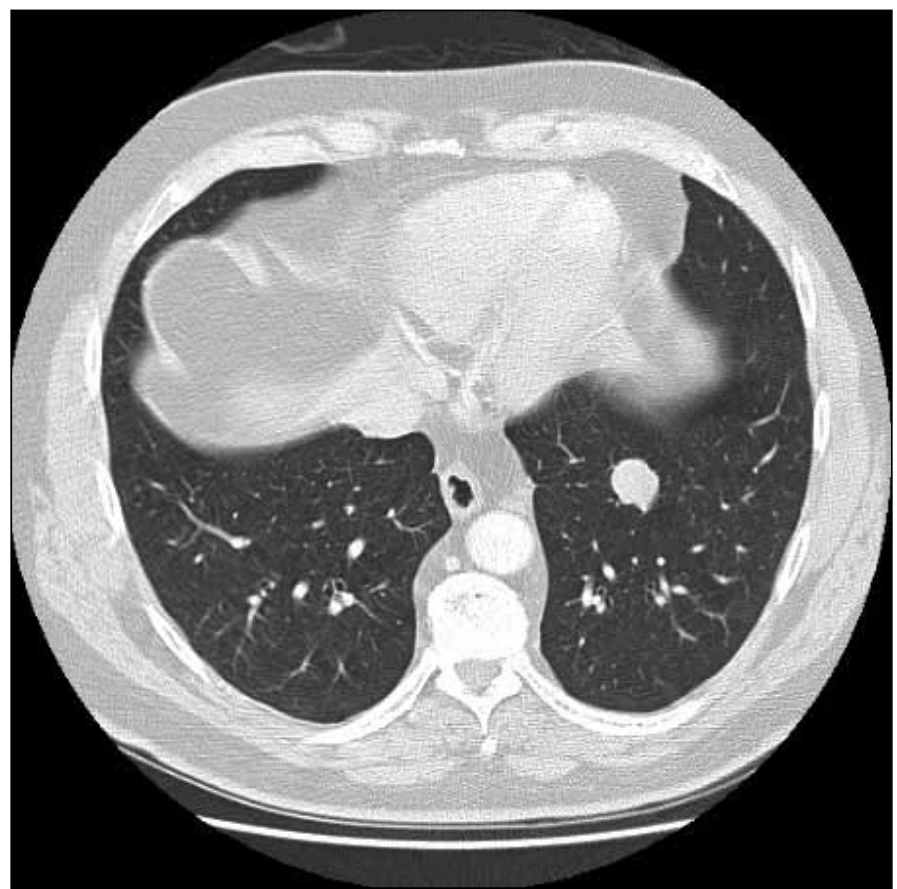

Fig. 2. Postoperative staging computed tomography scan demonstrating bilateral pulmonary metastasis.

3. Price EB, Mostoffi FK. Secondary carcinoma of the testis. Cancer 1957;10:592-5.

4. Johnson DE, Jackson L, Ayala AG. Secondary carcinoma of the testis. South Med J 1971;64:1128-30.

5. Hida T, Saga K, Ogino J, et al. Testicular swelling as the presenting sign of cutaneous malignant melanoma. J Eur Acad Dermatol Venereol 2006;20:351-3.

6. Datta MW, Young RH. Malignant melanoma metastatic to the testis: a report of 3 cases with clinically significant manifestations. Int I Surg Pathol 2008; 8:49-57.

7. Lowell DM, Lewis EL. Melanospermia: a hitherto undescribed entity. J Urol 1966;95:407-11.

8. Segelov E, Coates AS, McCarthy WH. Metastatic melanoma presenting at diagnosis as a testicular mass. Aust. NZ I Med 1992;22:701.

9. Tiltman AJ. Metastatic tumors in the testis. Histopathology 1979;3:31-7.

Correspondence: Dr. Muhammad Z. Aslam, Urology Office, Hambleton Wing, Friarage Hospital, Northallerton, DL6 IJG UK; fax: (0044)1609764833; mzaslam77@yahoo.com 